
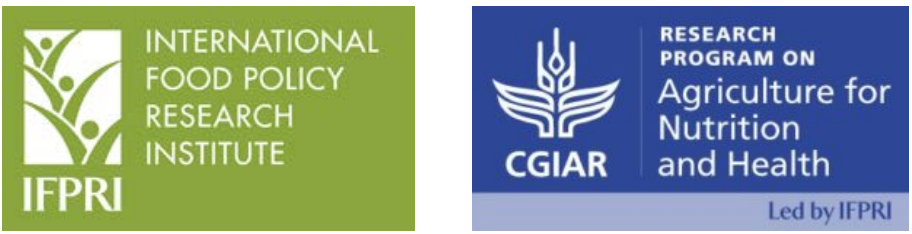

Independent Impact Assessment Brief

June 2019

\title{
EVALUATION STUDY OF THE IFPRI/A4NH RESEARCH PROGRAM ON DIET QUALITY AND HEALTH OF THE POOR
}

\author{
Jere R. Behrman and Shibani Ghosh
}

IFPRI's Poverty, Health, and Nutrition Division (PHND) and the CGIAR Research Program on Agriculture for Nutrition and Health ( $\mathrm{A} 4 \mathrm{NH})$ have conducted research since 2003 on the critical links between nutrition, health, and agriculture. This evaluation considers the impact of the work carried out through 2016, looking at the research strategy, engagement, capacity building, and impact on programs and policies and global dialogue. Findings suggest that the Diet Quality and Health of the Poor program has been successful in developing and sharing valuable research, knowledge, and data, and has brought new issues and approaches to partners and stakeholders. Through a range of projects, the program has effectively engaged with stakeholders, partners, and governments to support capacity enhancement and to help shape national interventions to improve nutrition. However, the original aspirations of the program have not been fully met and challenges in terms of communication and coordination indicate there is room for improvement.

\section{PROGRAM ORIGINS AND OBJECTIVES}

The Diet Quality and Health of the Poor research program-Global Research Program 24 (GRP24)—was launched in 2003 with the aim of generating a global understanding of the main drivers of current trends in dietary quality and dietary changes in order to identify effective policy levers to improve the dietary quality of the poor, reduce food insecurity and under- and overnutrition, and redirect the nutritional transition toward health, especially for the poor. GRP24 objectives were modified over time in view of the program's progress and limitations on funding.

In 2011, GRP24 was integrated with IFPRI's strategic research area on Promoting Healthy Food Systems, and when PHND led the establishment of the CGIAR Research Program on Agriculture for Nutrition and Health ( $\mathrm{A} 4 \mathrm{NH})$, which was designed to fill a research gap between agricultural development and its health and nutrition benefits, GRP24 became one of the program's four flagships: Integrated Programs and Policies for Nutrition. Research in the 2012-2016 period focused on programs and policies to improve dietary quality, nutritional status, and health of mothers, infants, and young children from impoverished environments, in particular interventions to improve nutrition during the early life "window of opportunity" - the 1,000 days from conception to when a child reaches 24 months-and on preventing, rather than treating, childhood undernutrition.

The work of the Diet Quality and Health of the Poor programs was carried out through some 140 projects, among which some of the most important were Alive \& Thrive, POSHAN, Transform Nutrition, Stories of Change, LANSA, and ARENA, with funding from a number of donors.

\section{EVALUATION}

This evaluation covers the impact of the research program from 2003 to the end of the first phase of the A4NH flagship program in 2016. Key questions we addressed include: Did IFPRI/A4NH have the right research strategy and make appropriate adaptations based on internal learning, stakeholder and partner inputs, and evolving circumstances? Has IFPRI/A4NH been effective in identifying, engaging, and supporting key partners and influencing its target audiences-other researchers, development program implementers, policymakers, and donors? How effective has IFPRI/A4NH been in increasing partner capacity, especially in developing countries, for identifying and analyzing data for better investment and policy decisions and designing, implementing, and evaluating multisectoral agriculturenutrition-health linked interventions? What has been the 
impact of the programs and policies that IFPRI/A4NH influenced?

To support this assessment, we consulted with researchers, clients, and other stakeholders. We selected India and Ethiopia for in-depth case studies in consultation with GRP24 and A4NH staff. These two countries accounted for a large share of the program's budget. In Ethiopia, PHND/GRP24 invested substantially in evaluation of the Productive Safety Net Program (PSNP) and in India, projects have focused largely on maternal and child nutrition. We conducted 39 interviews with participants, partners, and stakeholders associated with the work in these countries. Interview questions were tailored to the interviewees, but generally addressed experiences with the program, how well the program achieved its objectives, how well it used relevant approaches and methods, and to what extent it contributed to capacity building. Some caveats regarding the information gleaned from the interviews should be mentioned: the interviewees do not constitute a representative sample, and many had worked for GRP24, A4NH, or IFPRI; interviews were conducted in 2018, so answers are likely skewed toward more recent events; and not all interviewees were able to distinguish work under this program from similar or related work.

In addition, we reviewed numerous documents, including proposals, project abstracts, internal reviews, and research outputs, as well as measures of impact of the publications produced by the program.

\section{RESEARCH STRATEGY}

The primary focus of the Diet Quality and Health of the Poor programs was research. These programs were undertaken in the context of changing global challenges in nutrition and food security, including increasing recognition of the importance of nutrition during the first 1000 days, increasing prevalence of overweight and obesity, and substantial market changes. The appropriateness of research strategies, including adaptation to changing circumstances, is therefore integral to the programs' success.

Downloads of project papers, citations of publications, and presence on social media as measured by Altmetrics are useful measures of the appropriateness of research strategies. A number of PHND and A4NH projects generated substantial attention by these measures, including high rankings for several editions of the Global Nutrition Report, peer-reviewed literature reviews, and primary research articles.

Perceptions of participants, partners, and stakeholders provide another measure of research strategy appropriateness. The interviews raised several issues. First, while the agriculture-nutrition linkages research strengthened over time, especially after 2011, the programs were less successful in deepening research on the double-burden of nutrition, overweight and obesity, and urban populations, largely because neither the CGIAR and its donors nor most country governments were interested in funding this work. Second, context limited the acceptability of some research strategies, such as randomized controlled trials. In the case of PSNP evaluations in Ethiopia, for example, quasi-experimental methods were used rather than randomly assigning beneficiaries to the safety-net program. Third, the large scale of data collection required significant investment in data quality assurance, but also laid the foundation for more confident conclusions, and interviewees perceived that the data were of high quality. For example, in Ethiopia, the quality of the data and related findings allowed local researchers and policy professionals to bring key issues to the government's attention. The collection of consistent and comparable panel data was particularly important, allowing for a comparable baseline, midline, and endline for PSNP evaluation. In large, heterogeneous countries such as India, localized data can be essential for better investment and policy decisions, and development of Indian district-level profiles through the POSHAN project has been an important contribution.

\section{IDENTIFYING, ENGAGING, AND SUPPORTING KEY PARTNERS AND INFLUENCING THE TARGET AUDIENCE}

The interviews pointed to successes and failures in engagement. Continuity, including in-country residence and frequent visits, is critical to developing the strong relationships between researchers and stakeholders that allow for policy impact. Relations with donors are also critical to engage them with research strategies. The interviewees often were not aware of the role of PHND or A4 NH. However, stakeholders in Ethiopia and India reported that they were quite aware of IFPRI and the activities of programs with which IFPRI interacted or collaborated such as Alive \& Thrive, Ethiopia's PSNP, and POSHAN. For example, a former Ethiopian government official said he was familiar with Alive \& Thrive and suggested the project had driven thinking on infant and young child feeding (IYCF) in the country and that IFPRI research has been critical in moving Ethiopia forward with policy and strategy development. In India, stakeholders mentioned familiarity with the district profiling activities of POSHAN and IFPRI's positive relationship with Niti Aayog (the government think tank responsible for India's nutritional mission), and that IFPRI's papers and policy briefs are taken seriously. The generally positive perspectives on engagement were tempered by some challenges. For example, in India, where turnover of government personnel is frequent, the most stable partners were found to be in nongovernmental or "boundary" stakeholders rather than in governments. 


\section{ENHANCING PARTNERS' CAPACITIES}

Although the primary goal of the PHND and $\mathrm{A} 4 \mathrm{NH}$ programs was policy-relevant research, they also contributed to researchers' and stakeholders' capacities through "learning by doing" and more general support for research and evidence-based policies. Explicit efforts were made to build capacity for evaluations, including for example, short courses for district officials on nutrition and data in India and work with local universities, local investigators, and research assistants in IFPRI projects in Ethiopia. Work with Ethiopia's Central Statistical Agency (CSA) on evaluation of the PSNP generated capacity for panel data collection, electronic data collection, and ensuring data quality. A key impact of IFPRI's capacity building and training has been to change the language of evaluation-moving governments away from a focus on monitoring to evaluation, including systemic comparisons to generate counterfactuals. Despite these strengths in building partners' capacities, some interviewees suggested that it would have been better to have devoted more resources to direct capacity building, particularly of younger researchers.

\section{IMPACTS OF PROJECTS RELATED TO DIET QUALITY AND HEALTH OF THE POOR}

To evaluate the impact of the PHND and A4NH work in the absence of any quantitative estimates, we rely on narratives about policy influence and outcomes from stakeholder interviews and on data on the use of research outputs. As noted above, impact depends on both the quality of the research and continuity of involvement of policy and policy-related stakeholders.

In Ethiopia, researchers have worked closely with stakeholders on evaluation of the PSNP. Analysis of PSNP III showed that it was failing to have an impact on nutritional outcomes and that there were problems with getting funds to beneficiaries, both of which findings led to changes in the program design. Analysis and the lessons learned from the Alive \& Thrive program led to the integration of nutrition and social behavior change communication (SBCC) into PSNP IV, and SBCC materials are also now widely used in other contexts. Stakeholders reported substantial reliance of the PSNP on research and surveys conducted by IFPRI, noting the importance of the research in showing the impact of PSNP and helping with the redesign of the program for a shift toward greater nutrition sensitivity and building long-term resilience. In addition to the shift from monitoring toward evaluation, capacity for data collection increased, and the CSA led the evaluation for PSNP IV. Findings from Transform Nutrition in Ethiopia have supported work on nutrition-sensitive agriculture, multisectoral coordination, and water, sanitation, and hygiene (WASH) as well as nutritionspecific projects. In addition, the Lancet papers on nutrition-sensitive interventions have contributed substantially to policy discussions in Ethiopia, driving thinking on nutrition-sensitive agriculture, including by helping to identify appropriate interventions and developing indicators. Some efforts were less successful. For example, the Stories of Change project had interesting findings, but these do not appear to have been used much.

In India, IFPRI's work is widely cited and played a key role in bringing the disconnect between India's rapid economic growth and alleviation of undernutrition into public discussion. A number of projects are reported by researchers and stakeholders to have positive impacts. TANDI was an early project that led to the proliferation of agriculture-nutrition activities in India as well as globally. With findings from a maternal nutritional pilot, Alive \& Thrive helped develop a package of maternal nutritional interventions for the Ministry of Health, which is responsible for antenatal health. Niti Aayog's monitoring of Sustainable Development Goal indicators related to nutrition and health refers to IFPRI's work, and international organizations working in India make use of IFPRI studies. At the state level, Stories of Change is reported to have had significant traction, for example, leading the government of Odisha to request further policy engagement. POSHAN has developed district-level data that allows for an assessment of nutrition at a disaggregated level and is viewed as providing insightful data analysis and evidence on nutritional priorities. However, a few stakeholders felt that research was not leading to government uptake and policy change. And a problem arose with the Global Hunger Index, as many stakeholders perceived methodological problems with its analysis and ranking of nutritional status in India, posing a threat to the perceived quality of IFPRI research.

Quantitative indicators on use of GRP24 and $\mathrm{A} 4 \mathrm{NH}$ publications, including citations, Altmetrics, and downloads, provide another measure of how much the broader community is paying attention to, and being affected by, this research. Overall the numbers on use of this work are impressive, suggesting a broad reach to diverse audiences. Looking at $182 \mathrm{GRP} 24$ and $\mathrm{A} 4 \mathrm{NH}$ journal articles, there had been 2,689 citations as of early 2018. While some titles were never cited, one received 315 citations, and the median number of citations is 2.0 overall and 0.5 per year since publication. The top 10 were published across more than a decade (2002-2013), including five in nutrition journals, and the top one in an economics journal. Altmetrics provides a weighted count of the amount of attention a publication received in a range of online and other platforms (news stories, blogs, policy documents, and social media). Of 171 research outputs and external publications from GRP24 and $\mathrm{A} 4 \mathrm{NH}$, 29 research outputs and 55 external publications earned scores of 2 or more. The Altmetric score for the 2015 Global Nutrition Report is an order of magnitude greater 
than for any other item. Thirty Global Nutrition Reportrelated items-particularly the 2014, 2015, and 2016 reports and a complementary poster for 2015-accounted for 50 percent of total downloads $(193,745)$, out of 928 GRP24 and A4NH titles. Country and nutritional profiles accounted for another 27 percent.

\section{RECOMMENDATIONS}

The PHND and A4NH programs completed important research that has influenced both domestic partners and stakeholders and the international policy and research communities. These programs had both strengths and weaknesses, as revealed by the interviews and document reviews. The recommendations summarized here reflect the concerns of the interviewees and our own judgments.

1. Explore or revisit options for funding research on changing nutritional problems, such as overweight and obesity and dietary change.

2. Reconsider the balance between research and other activities to enhance implementation.
3. Consider using a wider range of methodological tools, such as structural economic models.

4. Develop studies not only at the national level but also at a disaggregated level for large, populous countries.

5. Engage stakeholders extensively, including in the choice of research topics to explore.

6. Improve communications by decoding research outputs into easily digestible briefs for decisionmakers and providing online learning models, online seminars for locally based stakeholders, and small grants for researchers and students.

7. Increase emphasis on formative research and on learning from less successful experiences.

8. Find ways to bring researchers together to ensure that the objectives of linking agriculture and nutrition are met.

Jere R. Behrman is William R. Kenan, Jr. Professor of Economics at the University of Pennsylvania. Shibani Ghosh is research associate professor at the Tufts University. 